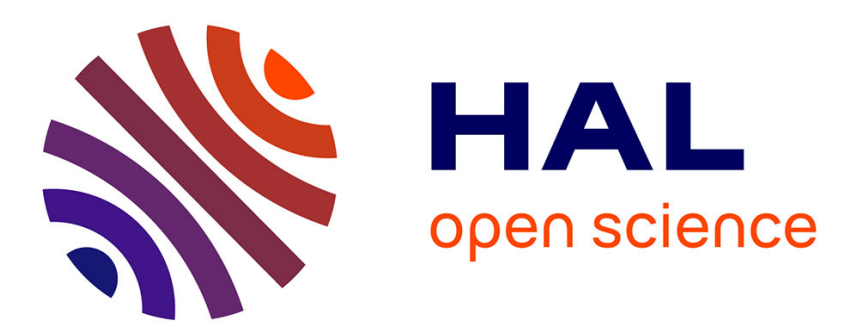

\title{
Le cérémonial diplomatique au temps des Mamelouks: Révélateur de la magnificence du sultan Sami Benkherfallah
}

\section{To cite this version:}

Sami Benkherfallah. Le cérémonial diplomatique au temps des Mamelouks: Révélateur de la magnificence du sultan. Revue Académique des Etudes Sociales et Humaines, 2018, 20, C (2), pp.12-24. halshs-01729752v3

\section{HAL Id: halshs-01729752 \\ https://shs.hal.science/halshs-01729752v3}

Submitted on 20 Sep 2018

HAL is a multi-disciplinary open access archive for the deposit and dissemination of scientific research documents, whether they are published or not. The documents may come from teaching and research institutions in France or abroad, or from public or private research centers.
L'archive ouverte pluridisciplinaire HAL, est destinée au dépôt et à la diffusion de documents scientifiques de niveau recherche, publiés ou non, émanant des établissements d'enseignement et de recherche français ou étrangers, des laboratoires publics ou privés. 


\section{الأكاديميت للدراسات}

\section{الاجتماعيت والإنسانيت}

\section{العدد - 20 جوان2018}

$$
\text { (ج) العلوه الاجتماعيت }
$$

Academic Journal of Social and Human Studies

\section{Etudes}

\section{Sociales \&. Humaines}

$N^{\circ} 20$ - June 2018

\section{C/ Social Sciences}


La " Revue académique des études Sociales et الأكاديميت للدراسات الإجتماعيت و الإنسانيت هي محلتة Humaines" est une publication scientifique académique avec comité de lecture international, à parution semestrielle en langues arabe, anglaise et française.

Elle est éditée par l'Université Hassiba Benbouali de Chlef, Algérie.

Thèmes

La revue se décline en trois séries

Série A : Economics and Law.

Série B : Letters and Languages.

-Série C : Social Sciences.

- Pour toute correspondance administrative,

s'adresser à :

Direction des publications, bloc recherche,

$3^{\text {ène }}$ étage, Université Hassiba Benbouali de

Chlef, BP 151, 02000, Chlef.

- Toute soumission d'article se fait exclusivement par

voie électronique à l'adresse :

Email : raesh_journal@univhb-chlef.dz

La Revue Académique des Etudes Sociales et Humaines est indexée par:

- PROQUEST, Michigan,USA

\section{ProQuest}

- EBSCO, Massachusetts, USA

\section{EBSCO}

- le Système National de Documentation en Ligne

$$
\text { دوليت سداسيت تعنى بنشر الإنتر }
$$

الأبحاث بِّ مختلف مجالات العلوم الإجتماعيتّ و الإنسانيت باللغات الثلاث : العربيت, الإنجليزيت , الفرنسيت. المجلت تصدر عن جامعتَ حسيبت بن بوعلي بالشلف.

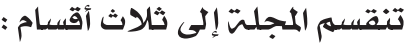

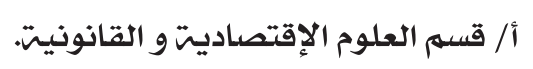
ب/ قسم الآداب و الفلسفتة. ج/ قسم العلوم الإجتماعية.

la librairie en ligne ALMANHAL

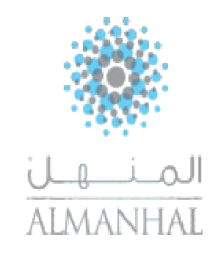

et l'AFRICAN JOURNAL ONLINE

$A J(s) L$

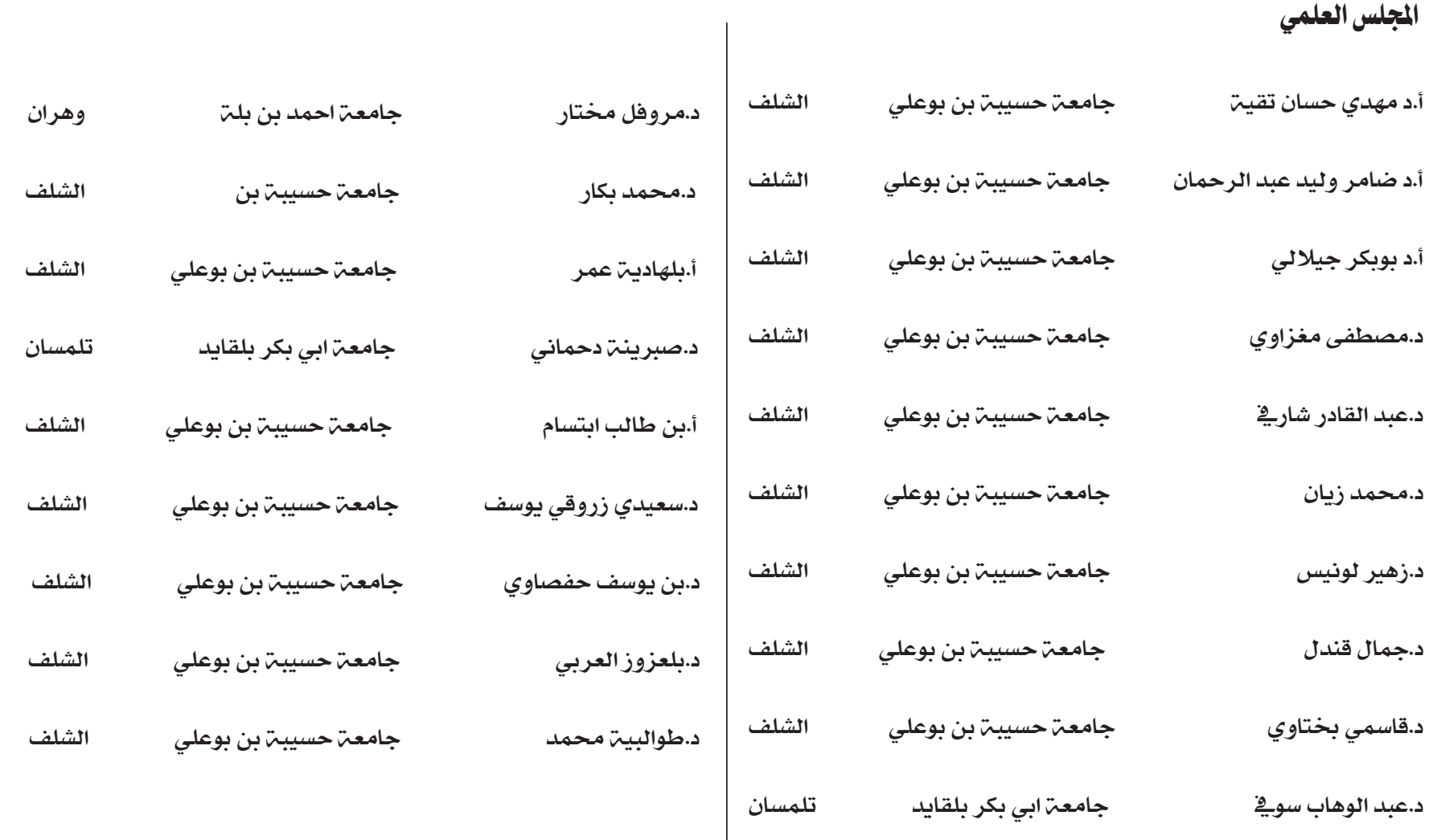

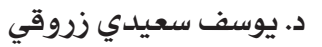

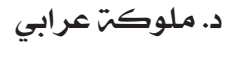


• تُعنى مجلت الأكاديميت ِِِ قسمها الثالث بالبحوث يِ العلوم الإجتماعيت. • تخضع البحوث المقدّمتة إلى المجلّت للتقويهم والتحكيم حسب الأصول المتبعت. • تُقبل البحوث باللغت العربيت والإنجليزيت والفرنسيت.

• يكون البحث المقدم للهجلت مستوفياً شروط البحث العلهي من حيث الإحاطت والاستقصاء والإضافت المعرفيت والمنهجيت والتوثيق وسلامت اللغت ودقت التعبير وعلى الباحث مـراعاة أسلوب البحث بحيث يكون موافقاً للقواعد اللغويّت. • ألا يتجاوز البحث المقدّم خمست عشر صفحت من الحجم العادي (A4). • يرفق البحث بملخص باللغتين العربيت والإنجليزيت بها لا يقلّ عن خمست عشر سطرا. • يشترط يِ عنوان البحث والكلمات الدالت أن تكون باللغتين العربيت والإنجليزيت. • ألا يكون البحث قد سبق نشره على أي نحو كان أو تم إرساله للنشر ٌِِ مجلت أخرى ويتعهد الباحث بذلك. • لا يجوز لصاحب البحث أو لأي جهت أخرى إعادة نشر ما نشر ِِّ المجلت أو ملخص عنه يِ أي كتاب أو صحيفت أو دوريتة إلا بعد مرور سنت على تاريخ نشره • يلتزم الباحث بعدم إرسال بحثه لأي جهت أخرى للنشر حتى يصله رد المجلت. • يلتزم الباحث بإجراء تعديلات المحكمين على بحثه وفق التقارير المرسلت إليه، وموافاة المجلت بنسخت معدلت ِِّ مدة لا تتجاوز 15 يوما. • لا يجوز للباحث أن يطلب عدم نشر بحثه بعد إرساله للتحكيه إلا لأسباب تقتنع بها هيئت التحرير. • يرفق البحث بهعلومات تخص الباحث، تتضمن اسمهه ودرجته العلميّتة، وتخصصه، ووظيفته والجهت التّي يعمل بها، رقم هاتفه وبريده الإلكتروني. • قرارات هيئت التحرير بشأن البحوث المقدّمت إلى المجلّت نهائيّتة، وتحتفظ الهيئت بحقها ِِِ عدم إبداء مبرّرات لقراراتها. (raesh_journal@univ-chlef.dz): • • • تكتب المادة العلميت العربيت بخط من نوع Simplified Arabic مقاسه 12 بمسافت 21 نقطت بين الأسطر، العنوان الرئيسي

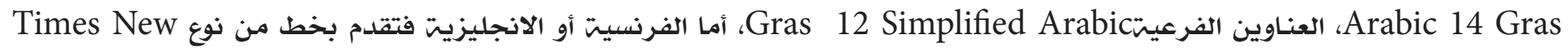
Roman • هوامش الصفحت تكون كمايلي: أعلى 02، أسفل 02، يمين 02، يسار 02، رأس الورقت 1.5، أسفل الورقت 1.25، حجم الورقت مخصص .$(16 \times 23.5)$ • ضبط الجداول و الأشكال مرقمت ومعنونت وفقا لهوامش الصفحت الآنفت الذكر ويستحسن أن تعد بالطريقت الآليت أي بالبرامج المخصصت لها. • يجب إثبات الهوامش مستوفاة يِّْ آخر البحث. • يجب أن يعرض رقم التهميث والإحالات يِ المتن بطريقت يدويت (1) (2) ... على أن تثبت يِّ نهايت المقال وفق الترتيب التالي: المؤلف، عنوان الكتاب أو المقال، عنوان المجلت أو الملتقى، الناشـر، البلد، السنـت، الطبعت والصفحت، وذلك وفق منهجيت الجمعيت الأمريكيت لعله النفس (APA). • البحوث المنشورة ِِِ المجلت تعبر عن آراء أصحابها، ولا تعبر عن هيئت التحرير أو المجلت. 


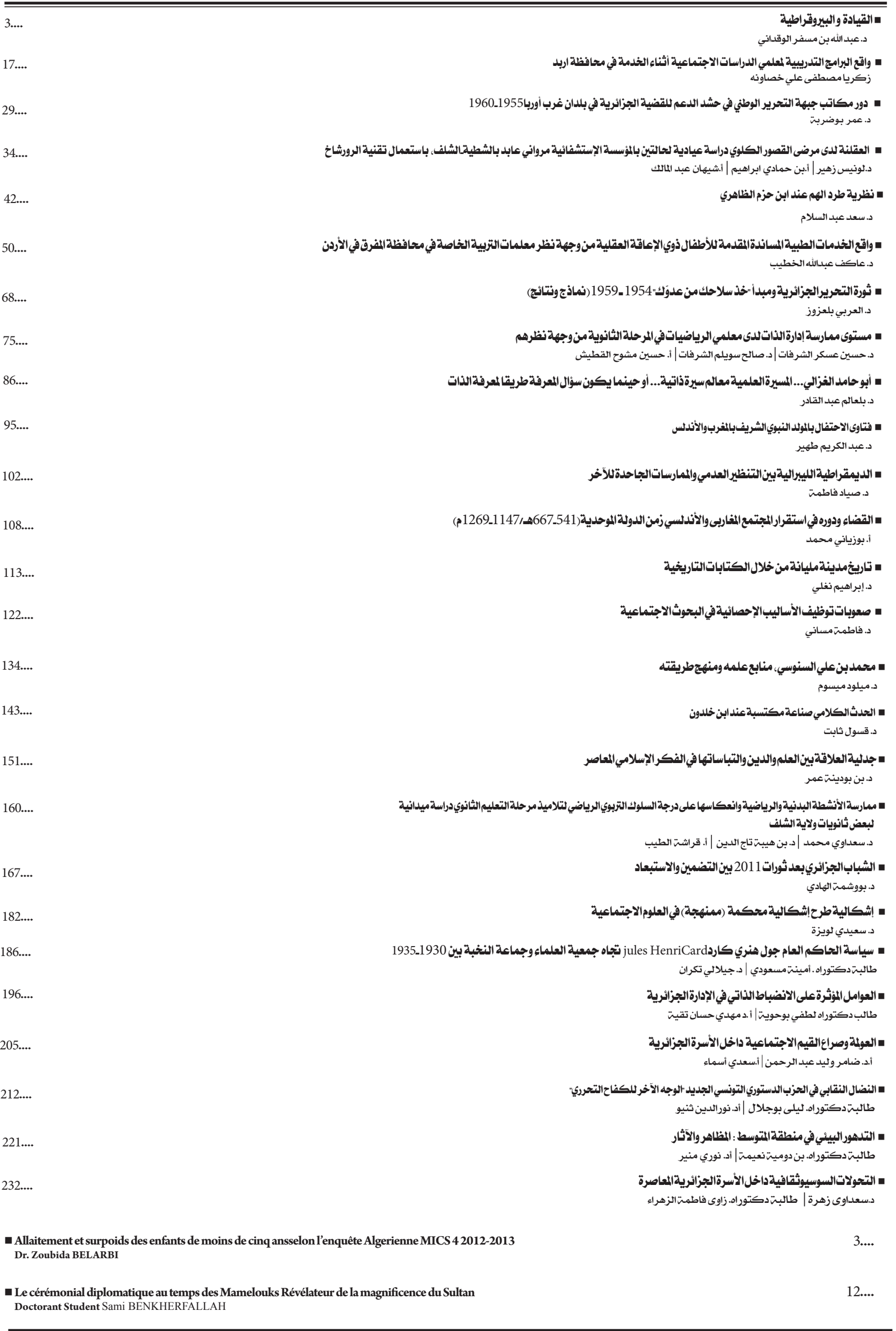




\title{
Le cérémonial diplomatique au temps des Mamelouks Révélateur de la magnificence du sultan
}

The diplomatic ceremonial during the Mamluk period : a revelant indicator of sultan's magnificence

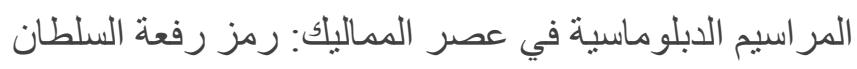

\section{BENKHERFALLAH Sami}

\section{Doctorant en Histoire}

Centre d'études supérieures de civilisation médiévale - University of Poitiers, France

Laboratoire des Études civilisationnelles \& intellectuelles - University of Tlemcen, Algeria

sami.benkherfallah@outlook.com

$$
\begin{aligned}
& \text { تعتبر هذه الورقة بمثابة مدخل إلى عالم الدبلوماسية في البلاد الإسلامية على عهد المماليك و هي تتناول بشكل خاص طريقة سير المراسيم التي }
\end{aligned}
$$

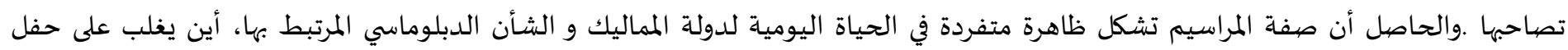

$$
\begin{aligned}
& \text { مراسيم استقبال و اعتماد الوفود الدبلوماسية إصرار على إبراز كل ما يرمز إلى قوة السلطان من حيث ثروته و رفاهية البلاط، كما أن القراءة }
\end{aligned}
$$

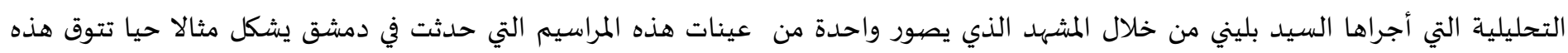

$$
\begin{aligned}
& \text { الورقة العلمية و تسعى إلى دراسته . } \\
& \text { الكلمات المفتاحية : الدبلوماسية - المماليك - المراسيم الدبلوماسية - البلاط المملوكي - التاريخ - تاريخ الفن }
\end{aligned}
$$

\section{Résumé}

Ce propos se veut être une introduction à la diplomatie au temps des Mamelouks et du cérémonial qui l'accompagne. Le cérémonial est un phénomène récurrent dans le quotidien des Mamelouks où la réception d'ambassade laisse s'exprimer le luxe et les richesses du Sultan et de sa cour. L'analyse du tableau de Gentille Bellini proposée dans cet article représente vraisemblablement une réception d'ambassade vénitienne à Damas.

Mots-clefs : Diplomatie - Islam médiévale - Mamelouks - Cérémonial - Histoire - Histoire de l'art.

\begin{abstract}
This presentation is an introduction to islamic diplomacy and its ceremonials during the Mameluk period. The ceremonial is a reccurent phenomenon in the everyday lives of the Mamluks, where the ambassy reception shows luxury and the riches of the sultan and its court. The analysis of Gentille Bellini's painting suggested in this article, which represents obviously an ambassy reception in Damascus, is an excellent exemple of it.
\end{abstract}

Keywords : Diplomacy - Medieval islam - Mamluks - Ceremonial - Art history. 


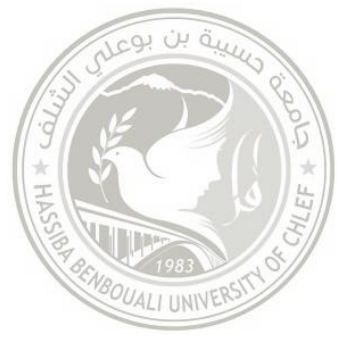

Les Mamelouks forment une dynastie d'esclaves ayant pris le pouvoir en Égypte après leur victoire sur les Mongols à Ain Jalût en 1260. Il s'agit d'un frein à leur expansion vers la Méditerranée. Les musulmans se sont trouvés dès lors sans calife, étouffé par les Mongols à Baghdâd quelques années auparavant (1258). Baybars, en fin stratège restitua le califat en Égypte, en intronisant un des derniers descendants du calife abbasside, ce qui lui conféra une légitimité politique qu'il ne possédait pas auparavant. Il s'en suit deux siècles et demi de pouvoir sur une partie du monde musulman, de rayonnement culturel, artistique et scientifique.

Le luxe est un élément essentiel de l'apparat des souverains d'Égypte et, plus particulièrement, de l'ère mamelouke. Ainsi, la cour a manifesté tout au long de la période un intérêt singulier pour la forme et l'apparence. Ces soldats, issus de régions âpres, vont se familiariser assez rapidement avec les richesses que leur procurent les terres d'Égypte et de Syrie.

Le cérémonial apparaît comme un véritable outil illustrant la hiérarchie sociale que le sultan veut imposer et faire paraître.

\section{LE CEREMONIAL A LA COUR}

Le cérémonial fait partie du quotidien des Mamelouks et de la société civile, se manifestant tout au long de l'année de manière spontanée (ou calendaire) comme lors de l'accession au trône d'un nouveau sultan ou de la réception d'un dignitaire étranger. De tels événements donnent ainsi lieu à un décorum minutieusement ordonné, dicté par des codes clairement établis, suivant un itinéraire préalablement défini. Mais leur but était précis : celui de distraire la population tout en mettant en évidence un rapport de force manifeste par le biais de la puissance militaire et de l'exhibition des richesses sultaniennes.

\section{LE CALENDRIER CEREMONIEL}

Il existe deux types de manifestation :

- Les manifestations calendaires : fêtes religieuses et fêtes civiles.

- Les manifestations ponctuelles (bashâ'ir) : annonces d'un événement important.

Les manifestations calendaires donnaient lieu à un cérémonial à la fois inspiré des calendriers solaire et lunaire.

Le calendrier solaire, dit civil :

- Cérémonial de la crue du Nil ${ }^{1}$.

- Cérémonial du changement de vêtements saisonnier ${ }^{2}$.

- Cérémonial ayant trait aux tournois de polo ${ }^{3}$.

Le calendrier lunaire, dit musulman :

- Salutations annuelles des dignitaires au sultan

- Mawlid (= fête célébrant la naissance du

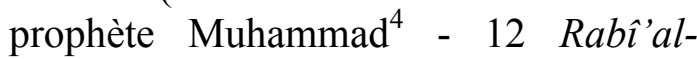
awwal)

- Départ et retour du pèlerinage à La Mecque donnant lieu à la cérémonie du mahmal $^{5}$ (10 dhû al-hidja)

- Lecture des hadiths ${ }^{6}$ de Bukhârî $\rightarrow$ Derniers jours du Ramadhân ${ }^{8}$

- 'Aid al-fitr (= fête de la rupture du jeûne) $\rightarrow$ Dernier jour du mois de Ramadhân ${ }^{9}$

-'Aid al-adha (= fête du sacrifice) $\rightarrow 10^{\mathrm{e}}$ jour de dhû al-hidja

- La prière du vendredi (salât al-jumu'a) ${ }^{10}$

Ces événements étaient l'occasion d'organiser de grandes manifestations militaires à cheval ${ }^{11}$ accompagnées de prouesses sportives $^{12}$. Ils se déroulaient dans de somptueux décors et les vêtements arborés ces jours-là étaient d'un grand luxe. Notons que, outre ces cérémonies habituelles, d'autres manifestations, dites spontanées, interviennent au fil de l'année comme la réception de hauts dignitaires étrangers.

Les manifestations spontanées :

- Cérémonies sultaniennes à la Citadelle

- Retours victorieux ${ }^{13}$

- Mariages

- Circoncisions 
- Naissances

- Réjouissances spontanées (chasses, jeux)

- Audiences ordinaires de la cour (audience des Mazâlim) ${ }^{14}$

- Réceptions d'ambassades et de dignitaires ${ }^{15}$

\section{LES RECEPTIONS D’AMBASSADES}

Les études menées sur la diplomatie connaissent un regain d'intérêt chez les chercheurs depuis quelques années ${ }^{16}$. La diplomatie a, de tout temps, assuré un pont culturel, commercial et politique entre espaces et religions.

Les réceptions d'ambassades concernent de près la vie de cour. Ainsi, le sultan choisissait les ambassadeurs parmi ses courtisans qui étaient les plus à même de le représenter. Cette mission exige des qualités propres au statut d'ambassadeur. Ces derniers pouvaient être issus de l'élite militaire (émirs), civile (hauts fonctionnaires), religieuse (patriarches melkites) ou marchande (négociateurs). La cour se trouve, de ce fait, sur le devant de la scène des échanges diplomatiques.

Le palais de la Citadelle, au cœur duquel les ambassadeurs étaient reçus, formait le cadre idéal d'exposition de toutes les richesses et d'un savoirfaire particulier en matière d'hospitalité ${ }^{17}$. Les réceptions d'ambassades représentaient la meilleure occasion de se rendre compte du faste des sultans et constituent, pour le sultanat, une réelle vitrine de sa magnificence et sont, pour le sultan qui s'y prépare scrupuleusement, un véritable enjeu diplomatique et relationnel.

L'accueil varie en fonction de l'interlocuteur reçu, on peut y observer cinq critères :

- La puissance, le statut et le poids de l'interlocuteur sur la scène diplomatique.

- La nature de la relation (bonne / mauvaise ?).

- Le critère de réciprocité.

- L'intérêt du déplacement et son enjeu.

- La connaissance de l'autre. Ainsi, verronsnous dans le cas de la venue d'une délégation vénitienne à Damas que le faste est moindre puisque les protagonistes en présence se connaissent déjà très bien.

\section{LA COUR, UN PREALABLE AUX NEGOCIATIONS}

\section{Éléments fondamentaux et normes diplomatiques}

Avant tout, il semble nécessaire de rappeler quelques éléments essentiels propres à la diplomatie musulmane et mamelouke.

Les réceptions avaient généralement lieu dans le grand Iwân de la Citadelle, alors subtilement décoré pour l'occasion ${ }^{18}$.

L'objet des négociations pouvait varier :

- La paix

- Le commerce

- Les prisonniers

- Les esclaves

- Les affaires épistolaires, culturelles ou scientifiques

- La simple courtoisie

\section{Concepts généraux}

La diplomatie musulmane s'est inspirée de celle en vigueur à l'époque du prophète Muhammad. Ainsi, les règles instaurées lors du développement du premier État musulman à Médine ${ }^{19}$ se sont perpétuées plusieurs siècles durant. Le monde se diviserait dès lors en deux espaces :

- Terre d'Islam (dâr al-Islâm : situation de « paix »).

- Terre de mécréance (dâr al-Kufr : situation de guerre ou de trêve).

Les relations devant être régies entre ces deux parties relèvent d'un état de guerre continuel, selon deux cas de figure distincts :

- Le territoire de la guerre (dâr al-harb).

- Le territoire de l'accord (dâr al- 'ahd).

Ce que l'on appelle « paix » en Occident chrétien est en réalité une trêve $\left(h u d n a^{20}\right)$ dans le monde musulman.

Quatre types de traités existent :

- Traité de soumission

- Traité d'alliance

- Traité de commerce (lié à la trêve)

- Traité de trêve (lorsque la paix ne peut être maintenue) 
Figure 01 : Schéma récapitulatif

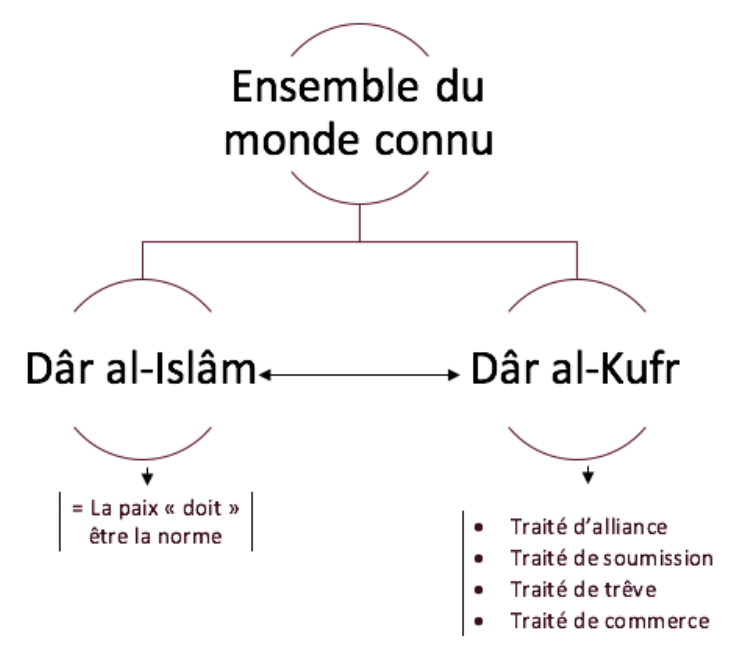

Dès lors, ces éléments constituent la base de la diplomatie islamique qui s'est complexifiée au fil $\mathrm{du}$ temps. Les fonctionnaires de la chancellerie avaient en charge l'écriture des correspondances entre souverains. Certains d'entre eux nous ont fait parvenir des manuels de chancellerie à l'image d'alQalqashandî $^{21}$ où est définie la forme des actes et du protocole. Des émissaires, nommés safîr (plu. safirûn) avaient pour mission, quant à eux, de transmettre des lettres diplomatiques. A noter que la carrière d'ambassadeur n'existe pas, seul le temps de la mission lui assure ce statut.

\section{LA GUERRE SUBORDONNEE A LA DIPLOMATIE}

La guerre régit l'essentiel des relations alors entretenues. La tradition diplomatique musulmane veut qu'un souverain prévienne son adversaire avant de l'affronter et l'incite à se soumettre à son autorité. Ainsi, durant les premiers temps de l'Islam, les généraux arabes chargeaient leurs armées nouvellement converties de prévenir leurs ennemis avant de les attaquer, leur demandant d'accepter l'islam comme religion ou de conserver la leur en échange du paiement d'un tribut. Si les Mamelouks avaient abandonné cette tradition, certains souverains se sont réappropriés cette pratique comme ce fut le cas lors de leur confrontation avec les Mongols de Perse. Il est possible d'affirmer sans ambages que la guerre induit, en partie, les relations diplomatiques des puissances musulmanes de cette époque, puisque lorsqu'elles n'étaient pas en guerre, elles s'apprêtaient à le devenir. En effet, chaque puissance musulmane se devait de maintenir ses frontières ou de les étendre.

La guerre implique, par défaut, le jihâd (la guerre sainte). Cette notion est réglée par une juridiction complexe. Ibn Taymiya ${ }^{22}$ apparaît comme l'un des plus grands théologiens à avoir étudié la question. Ayant vécu à l'époque mamelouke, il porte à notre connaissance nombre d'éléments concernant la diplomatie entretenue par les Mamelouks à l'égard des Mongols, ces derniers ayant envahi les territoires mamelouks ${ }^{23}$ entre 1299 et 1303. Dans une lettre ${ }^{24}$, il interpella le sultan Muhammad b. Qalâwûn ${ }^{25}$ sur la nécessité de déclarer le jihâd à leur encontre ; Ibn Taymiya considérait les Mongols comme des polythéistes faussement convertis à l'islam ou du moins pratiquant un islam déviant.

Le jihâd pouvait également être mené à l'intérieur des frontières. Dans ce cas les individus visés étaient ${ }^{26}$ :

- Les rebelles (bujât - mutamaridûn)

- Les apostats (ahl al-ridda)

- Les brigands (muhâbirûn)

Selon al-Mâwardîî (450H/ 1058) dans les ahkâm al-sultâniyya, la guerre sainte pouvait être menée contre les polythéistes (mushrikûn), les hérétiques et les brigands. Deux termes désignaient alors la guerre. D'une part, le jihâd qui désignait la guerre sainte et, d'autre part, le qitâl qui peut être traduit par « tuerie » ou «affrontement mortel ${ }^{28}$.

Le jihâd prend une toute autre tournure au temps des Mamelouks, dont la seule légitimité était tirée de la guerre. Sa proclamation relevait de la responsabilité du calife, qui n'avait toutefois plus qu'un rôle symbolique depuis le $\mathrm{X}^{\mathrm{e}}$ siècle, voilà pourquoi la charge avait été déléguée au sultan. Ces prérogatives sont sans cesse rappelées dans les titulatures des sultans, tout comme dans leurs discours, ainsi que le rappelle le calife :

«Sachez, ô hommes, que l'imâmat est l'une des prescriptions obligatoires de l'Islam, que le jihâd est prescrit à tous les hommes, mais que l'étendard de ma guerre sainte ne saurait être brandi que si l'union règne parmi les serviteurs de Dieu [...] Ce sultan, al-Malik al-Zâhir, le seigneur illustre, savant, juste, le 
combattant de la foi, le gardien des frontières, le pilier du monde de la religion, s'est dressé pour défendre l'imâmat, qui ne comptait plus qu'un petit nombre de combattants. Il a dispersé les armées infidèles qui avaient déjà pénétré au centre de nos pays. Grâce à ses soins, le serment de fidélité a été prêté universellement et la dynastie abbasside qui a trouvé de nombreux soldats.» 29

\section{LE COMMERCE}

L'histoire témoigne de nombreux cas où l'intérêt commercial a pris une place importante dans les clauses évoquées lors des échanges diplomatiques et cela dans le but d'élaborer des marchés ou de régler des différends entre commerçants ${ }^{30}$. Les khawajjâ $^{31}$ représentaient les intérêts marchands du sultan et plus précisément en ce qui concerne le commerce des esclaves ${ }^{32}$. Quant aux négociants, on leur préfère le terme de kârimî. Certains marchands semblent même être de véritables partenaires du sultan, en somme des interlocuteurs privilégiés de la diplomatie sultanienne.

Sur place, divers aménagements étaient réservés aux étrangers, marchands ou au corps diplomatique :

- Les caravansérails : les voyageurs y logent et stockent leurs marchandises

- Les consulats : représentation diplomatique

\section{LA RECEPTION, LA CORRESPONDANCE ET LE} PERSONNEL

Le statut d'ambassadeur ne constituait pas un métier à part entière. De fait, celui qui était investi de cette mission devait posséder les qualités propres à celle-ci. Ce pouvait être un haut dignitaire religieux, musulman ou chrétien, voire un haut fonctionnaire de l'élite civile ou militaire. Prenons ici un exemple : Ibn Khaldûn ${ }^{33}$ était un homme de cour qui travailla pour les plus grands royaumes de l'ensemble du monde musulman pour lesquels il a souvent rempli la fonction d'ambassadeur. Il fut notamment envoyé auprès des têtes couronnées chrétiennes d'Espagne au service de souverains d'Afrique du Nord. Durant son séjour au Caire, le sultan l'a nommé ambassadeur alors qu'il occupait la fonction de grand cadi malikite, dans le but d'aller négocier auprès de Tamerlan qui venait de ravager Alep.

L'ambassadeur devait être un homme pieux, de bonne réputation, honnête, regroupant toutes les qualités morales du «bon musulman ${ }^{34}$. Il s'agit, bien évidemment, de la norme, dans la pratique il en va autrement. L'histoire compte plusieurs ambassadeurs malicieux, hypocrites, antipathiques et ne portant aucun intérêt aux prérogatives religieuses.

L'utilisation d'interprètes, par ailleurs, est essentielle. Ils sont appelés drogmans et sont incontournables dans toutes négociations quelle qu'en soit la nature (politique, commerciale, amicale). Nombreux interprètes se sont convertis à l'Islam, leur double culture est, pour eux, un atout indéniable. Les États les moins puissants du dâr alIslâm ont dû recourir à des interprètes nonmusulmans pour répondre aux exigences du protocole diplomatique. Ainsi, l'essor du commerce avec l'Europe a engendré une demande de traducteurs au service de la chancellerie plus importante. De nombreux étrangers poussés par l'esprit d'aventure ont ainsi fini par intégrer le personnel diplomatiquedes souverains musulmans.

Les délégations diplomatiques en mission pouvaient rester plusieurs semaines, plusieurs mois, voire même plusieurs années. Les ambassadeurs devaient patienter longuement avant d'être admis en audience auprès du souverain ${ }^{35}$ et ils devaient, au minimum, parler la langue du pays dans lequel ils se rendent ou du moins disposer de traducteurs.

Bien que le métier d'ambassadeur n'ait pas existé en tant que tel, certains ont occupé cette fonction à plusieurs reprises devenant ainsi de véritables professionnels de la diplomatie, tel a été le cas d'Ibn Khaldûn ou encore de l'émir Fares ad-dîn al'Amidî qui, au moins trois fois, remplit cette mission auprès de la cour byzantine. Notons d'ailleurs que durant près de deux ans il a été retenu à Constantinople avant d'être rejeté par Baybars à son retour pour non-respect du protocole.

Les échanges entre souverains étaient influencés par les situations changeantes en Méditerranée. En revanche, la composition des délégations, elle, demeurait inchangée :

- L'ambassadeur(s) 
- Le personnel diplomatique (interprètes, scribes, notaires, religieux, commerçants, émirs, professionnels de la diplomatie)

\section{RECEPTION DES AMBASSADEURS A LA COUR} MAMELOUKE : ITINERAIRE

Leur venue est annoncée aux frontières par les gouverneurs locaux via un système postal très efficace développé à cette époque ${ }^{36}$. Les ambassadeurs restent aux frontières dans l'attente de la réponse du souverain. Une fois obtenue l'autorisation d'entrer sur le territoire, ils sont escortés jusqu'à la cour du souverain qui leur accordera une audience dans la salle du grand Iwân apprêtée pour l'occasion. Ornée d'une multitude de décorations et autres objets de valeur, elle donne même à voir des automates servant le thé ou jouant de la musique ${ }^{37}$. Ces ambassades, porteuses de messages écrits et oraux dans un premier temps, colportaient, à leur retour de mission, des informations concernant la langue, la culture, la puissance économique, l'armée et les coutumes des contrées parcourues. Elles rapportaient, en outre, les cadeaux transmis par le sultan qui permettaient d'exposer la richesse et la puissance du donateur.

\section{LE DON, UN CRITERE D'APPRECIATION}

Les cadeaux entrent dans la propagande des souverains et varient en fonction du message que l'on veut transmettre. Il s'agissait, généralement, de propositions d'amitié, de trêve, d'échanges de prisonniers ou encore de l'annonce de l'avènement d'un nouveau souverain.

Le don apparaissait comme l'un des plus importants aspects du cérémonial diplomatique, les membres de la cour appréciaient s'offrir des cadeaux en guise de gratitude. Notons, ici, deux éléments à prendre en considération :

- La lettre diplomatique ${ }^{38}$ (son contenu, la titulature, le poids du sceau, les matériaux utilisés, la qualité du support, l'ornementation, le style calligraphique et la valeur de l'étui).

- Les dons (la nature, la qualité et l'importance du don).
Les dons et le protocole diplomatique sont à " gine de la rédaction de recueils diplomatiques 17 Itres œuvres littéraires indépendantes. Le Kitâb al-nadâyâ wa al-tuhaf dit « Livre des dons et Trésor » écrit par Ibn al-Zubayr au XIe siècle en est un excellent exemple.

Quatre types de dons sont pratiqués à la cour (réguliers, ordinaires, extraordinaires et occasionnels). Le don et son corollaire, le contredon, avaient cours principalement entre le sultan et ses courtisans, même si une catégorie singulière était réservée en partie aux réceptions d'ambassades (dons occasionnels). Le sultan étant le garant des richesses sultaniennes, il se devait d'être généreux :

Don/contre-don régulier du courtisan envers le $\operatorname{sultan}^{39}$

Don/contre-don ordinaire du sultan à l'égard de ses courtisans

Don/contre-don extraordinaire

Don/contre-don occasionnel : lors de la réception d'ambassades

\section{Qu'échangeait-on lors des réceptions d'ambassades?}

Les dons étaient variés :

- Animaux : chiens, lions, faucons, chevaux, éléphants, girafes, panthères.

- Hommes non-libres : esclaves, prisonniers ou semi-esclaves (= eunuques).

- Armes : épées, lances, poisons.

- Textiles ${ }^{40}$ : tissus, étoffes tissées de fils d'or et/ou de soie.

- Proposition matrimoniale ${ }^{41}$.

- La lettre qui, en soi, pouvait être considérée comme un don 49 .

- Le sceau ${ }^{42}$.

- Médicaments.

- Manuscrits.

- Mobiliers : coffres, coupes, meubles.

- Euvres d'art : savoir-faire artisanal, tableaux, cristaux taillés, orfèvrerie, vaisselle

- Monnaies : or ou argent.

- Bijoux : perles, pierres, médaillons, métaux précieux.

- Transfert de savoirs : culinaires, intellectuels. 
L'appréciation du luxe était, globalement, la même à travers toutes les cours du monde. Ainsi, une certaine homogénéité est observée quant aux dons intervenant dans le contexte diplomatique. Les souverains appréciaient les cadeaux rares, précieux, ceux que l'on ne trouvait pas dans leur royaume.

Les offrandes diplomatiques s'inscrivent dans des relations de rivalité qui se tissent entre celui qui donne et celui qui reçoit et ce, dans l'optique de surenchérir au fil des dons. L'important étant de se surpasser dans l'extravagance et le luxe.

\section{Quelle dimension ces dons avaient-ils?}

Le don traduit trois dimensions : matérielle, économique et politique.

- Matérielle : l'intérêt de l'objet et son utilité en soi.

- Économique : les cadeaux diplomatiques engendrent une dynamique artisanale (travail de la soie, des pierres, de l'or).

- Politique : le cadeau était synonyme de paix, d'une part, et valorisait, par ailleurs, le prestige du souverain qui le recevait.

Il s'agissait, finalement, d'offrir un cadeau que le bénéficiaire ne pourrait égaler ${ }^{43}$. La rareté du don était un critère d'appréciation essentiel.

$* * *$

Les pigeons voyageurs permettaient une communication rapide et efficace entre les autorités locales et centrales du Caire qui annonçaient, rappelons-le, l'arrivée des délégations diplomatiques une fois celles-ci en vue. Toutefois, au vu de la taille du message, le contenu et la forme de la lettre se devaient d'être concis. Le tolédan Pero Tafur nous a fait parvenir un témoignage de ce type de communication en ces mots (1436) :

«J'ai vu là-bas pour la première fois les colombes qui portent les lettres dans une plume de la queue. On les convoie du lieu où elles ont été élevées vers un autre, on leur met la lettre et on les lâche : elles regagnent alors leur domicile. On fait cela pour connaître rapidement les nouvelles des gens qui viennent par terre et par mer, afin que les habitants ne soient pris par surprise, eux qui vivent sans forteresse ni mur ${ }^{44}$.

Ainsi, deux types de document coexistaient :

- La lettre de créance

- La lettre diplomatique

Tout n'est pas mentionné dans la lettre, une partie de l'information demeure orale. De fait, elle devait davantage servir à la correspondance interne, recourant de nombreux points de relais pour les grandes distances. Contrairement à celui en usage chez les Abbassides, l'utilisation des pigeons voyageurs était l'exclusivité du sultan et apparaissait comme l'un des éléments de distinction hiérarchique au sein de l'élite mamelouke ${ }^{45}$.

Ces courriers étaient privilégiés surtout en temps de guerre ${ }^{46}$. Ainsi, les forces mongoles ont été informées $\mathrm{du}$ rassemblement des troupes mameloukes dans l'ensemble du sultanat par ce biais ${ }^{47}$.

En terre chrétienne, les musulmans pouvaient jouir de trois statuts :

- Musulmans d'autres régions au service du souverain.

- Ambassadeurs.

- Prisonniers.

DOCUMENTS DIPLOMATIQUES ET MANUELS DE CHANCELLERIE

Le document diplomatique est généralement transcrit en plusieurs langues, celle de l'expéditeur et celle du destinataire. Les deux versions pouvaient se suivre sur le même support ${ }^{48}$. Les régions du dâr al-Islâm ne possèdent que très peu d'archives mais de nombreuses informations nous sont parvenues grâce aux manuels de chancellerie servant de modèle pour la composition d'autres traités comme le manuel de Qalqashandî, Subh al$a$ 'shâ ${ }^{49}$ ou celui de Shihâb al-Dîn al-'Umarî qui rédigea un texte à l'usage des scribes ${ }^{50}$. Traditionnellement, recourir à de lourdes bureaucraties ou à des chancelleries complexes n'était pas d'usage, les négociations orales ${ }^{51}$ étant davantage usitées. 
Une titulature spécifique était attribuée à chacun des souverains dans les manuels de chancellerie. Ils étaient alors classés selon leurs zones géographiques d'influence et leur confession. Al-'Umarî et al- Qalqashandî consacrent un chapitre exclusif "aux rois des infidèles $»^{52}$. En voici un extrait, tiré d'une traduction issue du manuel d'al-Qalqashandî montrant la façon dont sont nommés et classés les souverains chrétiens.

« Section II : Sur le protocole à observer par les rois des territoires égyptiens dans leur correspondance avec les rois des infidèles [...] Sachez que les rois infidèles des Grecs, des Francs, des Géorgiens, des Ethiopiens, et autres sont tous chrétiens [...]61 Section IV : Correspondances avec les princes infidèles dans la partie septentrionale de Rome et de Frandja, nationalités diverses mais toutes de confession melkite.

Correspondance avec le Pape.

Correspondance avec le roi des Romains, le souverain de Constantinople...

Correspondance avec les régents de Gênes... Correspondance avec le régent de Venise... Correspondance avec la souveraine de Naples... "

ÉTUDE D'UNE RECEPTION D'AMBASSADE VENITIENNE A DAMAS AU REGARD DU TABLEAU SUPPOSÉ DE GENTILE BELLINI ${ }^{53}$

\section{DESCRIPTION DE L'EEUVRE}

L'œuvre présentée est révélatrice des relations ayant existé entre Venise et l'Orient, mettant en évidence les fastes de la cour mamelouke. De nombreux rapports de rencontres diplomatiques nous sont parvenus, mais les illustrations faisant état de cela sont trop peu nombreuses. L'œuvre exposée représente la réception d'une ambassade vénitienne à Damas qui aurait eu lieu entre 1488 et 1511. Elle est conservée au musée du Louvre à Paris sous le numéro d'inventaire 100. Il s'agit d'une peinture à l'huile sur toile, peinte à Venise en 1511. Le tableau, mesure $201 \mathrm{~cm}$ en largeur sur 175 en hauteur, et a été rapatrié d'Istanbul au XVII siècle, enrichissant de fait les collections de Louis XIV. Longtemps cette toile a été sujet à controverse quant au lieu peint (Istanbul ? Le Caire ? Damas ?) et aux protagonistes représentés (Vizir ? Sultan ?). Tantôt attribuée à la cour ottomane tantôt aux Mamelouks, elle a fait l'objet de nombreux débats aux divergences non moins importantes ${ }^{54}$. Enfin, s'agissant de l'artiste, la paternité de l'œuvre, elle aussi, demeure très controversée : si l'auteur n'est pas Gentile BELLINI, la peinture est du moins de la main de l'un de ses élèves. Gentile BELLINI est un peintre italien du $X V^{\mathrm{e}}$ siècle, né en 1429 et décédé en 1507 à Venise, appartenant à une famille de peintres remarquables ${ }^{55}$. Notons qu'il fut l'un des peintres les plus talentueux de son époque ayant même été désigné pour réaliser le portrait du sultan Mehmed II $^{56}$. Venise étant une plaque tournante de l'apparat commercial méditerranéen, la cité put s'exporter au-delà de l'Italie, vers l'Anatolie, la Grande Syrie et en Égypte. Son statut de chef de file commercial lui a permis d'exercer une hégémonie économique (grâce au commerce des épices) sur l'ensemble de la Méditerranée durant des siècles. Cette position a engendré des relations diplomatiques privilégiées avec les états musulmans d'Orient, au premier plan 
desquels celui des Mamelouks.

De nos jours encore, quelques doutes subsistent quant à l'interprétation du tableau. Dans un premier temps, on y voyait la représentation d'un vizir à la cour ottomane, puis le sultan Mehmet II lui-même recevant un dignitaire vénitien à Istanbul $^{57}$, l'attribuant alors avec certitude à Gentille BELLINI qui s'était rendu sur ces terres en $1479^{58}$. Cette version avait induit une réflexion de Marco BOSCHINI, un théoricien de l'art et peintre italien du XVII ${ }^{\mathrm{e}}$ siècle. D'après lui, la bâtisse à l'arrière-plan ne pouvait être que la basilique Sainte- Sophie ${ }^{59}$. Cette hypothèse avait été acceptée jusqu'à ce que l'avis de Charles SCHERFER devienne la plus plausible.

\section{INTERPRETATION}

Charles SCHERFER y voit, en effet, la réception d'une ambassade à la cour mamelouke, démontrant qu'il ne pouvait s'agir d'un événement se déroulant à Istanbul ${ }^{60}$. Il y voyait plutôt l'Égypte et la Citadelle du Caire mettant en scène le sultan mamelouk Qansuh al-Ghawrî ${ }^{61}$ recevant un haut dignitaire vénitien $\mathrm{du}$ nom de Domenico TREVISANO, alors procurateur ${ }^{62}$. Cette version est plus proche de la réalité tout en restant défaillante. De fait, cette ambassade a bien eu lieu toutefois, l'identité de l'auteur initialement évoqué devient incertaine et source de questionnements. Cette ambassade s'est bien rendue sur les lieux en 1512, seulement Gentile BELLINI meurt en $1507^{63}$. Dès lors, cette interprétation ne peut s'avérer concluante, nous pouvons pour cela analyser la description donnée par l'un des témoins oculaires ${ }^{64}$ de cette ambassade pour s'en rendre compte :

«Nous entrâmes enfin dans une vaste cour à ciel ouvert, bien plus grande que la cour de Saint-Marc. Le Soudan était assis au fond de cette cour, sur un mastaba élevé de deux pieds au-dessus du sol et couvert de velours vert. Il avait sur la tête une très haute coiffe, avec deux cornes hautes d'un demi- bras. Le derrière de cette coiffe était formé d'une toque à côtes dont je ne saurais donner la description. Il était vêtu d'une robe de coton, sur laquelle il avait enfilé une autre robe de camelot vert foncé et il était assis, les jambes croisées, à la manière des tailleurs. A sa droite étaient placés son sabre et son bouclier. Non loin de lui et à sa droite se tenaient vingt personnes environ; toutes étaient vêtues de blanc et avaient une coiffure semblable à la sienne ». ${ }^{65}$

Cette description aurait pu correspondre à ce tableau étant donné les nombreuses similitudes, toutefois certains détails demeurent inexacts ${ }^{66}$ :

- L'espace décrit est beaucoup moins grand que celui de Saint-Marc.

- Le mastaba est recouvert d'un tissu rouge et non vert.

- Le chapeau que porte le personnage principal comporte quatre cornes et non deux.

- Le sabre et le bouclier sont absents.

- Les nombreux mamelouks présents ne sont pas tous vêtus de blanc et portent une taqqiya sur la tête.

De nombreux éléments permettent ainsi de définir le cadre dans lequel se déroule la scène. D'une part, les éléments architecturaux apportent quelques informations à propos d'un monument toujours existant : la mosquée des Omeyades. S'ajoutent à ceux- ci d'autres éléments, vestimentaires en l'occurrence, typiques de la région de la Grande Syrie, notamment en ce qui concerne les dames au second plan arborant le tantur, et les vêtements portés par les paysans originaires de la région de Naplouse au premier plan à gauche indiquent que cette scène se passe dans la région du bilâd alShâm ${ }^{67}$.

Figure 02 : Femmes vêtues du tantur

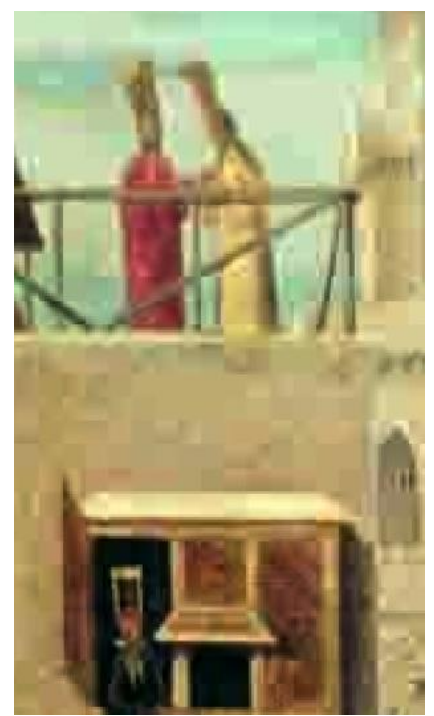


La rencontre, modeste, intervient entre individus se connaissant déjà très bien puisque, habituellement, la réception aurait lieu dans une salle dédiée à cet effet, appelée salle d'Audience.

\section{MISE EN SCENE : LA COUR AU COMPLET}

Le peintre a représenté une véritable scène de cour. L'individu coiffé d'un grand couvre-chef est le personnage principal auquel s'adresse la délégation, probablement s'agit-il d'un émir. Il est assis, jambes croisées à la turque ${ }^{68}$, sur un mastaba ${ }^{69}$ de velours rouge, la main posée sur ses genoux. Ses pieds sont recouverts d'un tissu vert kaki. À ses côtés, quatre religieux (sûrement des cadis) sont reconnaissables au turban blanc qu'ils arborent. Près de l'émir, deux d'entre eux se tiennent debout, les deux autres étant assis ${ }^{70}$. Ils représenteraient les cadis des quatre écoles juridiques. En face, proche de la délégation vénitienne, se tient un homme de dos s'adressant à l'émir, c'est l'interprète (drogman), un personnage incontournable des rencontres diplomatiques. Deux groupes de personnages portant des tâqîya-s rouges, constituent l'élite militaire des Mamelouks, ils sont reconnaissables à leurs vêtements. La délégation vénitienne est constituée, quant à elle, de cinq personnes habillées de la même manière, dont une se distingue de par sa toge rouge écarlate ${ }^{71}$. Souvent fait de laine, ce vêtement, emprunté à la Rome antique, est réservé aux doges de $V_{\text {enise }}^{72}$. A l'époque mamelouke, le vêtement était un élément de distinction sociale. Et grâce à ces quelques observations vestimentaires, nous pouvons sur ce tableau distinguer plusieurs catégories qu'il est possible de classer selon le genre, l'origine sociale, le rang ou la provenance géographique.

Dans le cadre de leur quête des épices et la lutte menée contre les Portugais, les Vénitiens et les Mamelouks entretenaient d'excellentes relations ${ }^{73}$. Voilà pourquoi Jean SAUVAGET pense qu'il s'agit de commerçants résidant sur place ${ }^{74}$. La modestie protocolaire relevée atteste parfaitement du fait que les protagonistes en présence se connaissent de longue date.

Les nombreux animaux et l'environnement verdoyant apportent une touche exotique à l'œuvre. La Syrie était connue pour ses nombreux arbres fruitiers dont le sultan mamelouk raffolait. Il en faisait importer de nombreuses variétés pour les planter dans les jardins de la Citadelle, lieu de résidence de la cour ${ }^{75}$.

Figure 03 : Délégation vénitienne

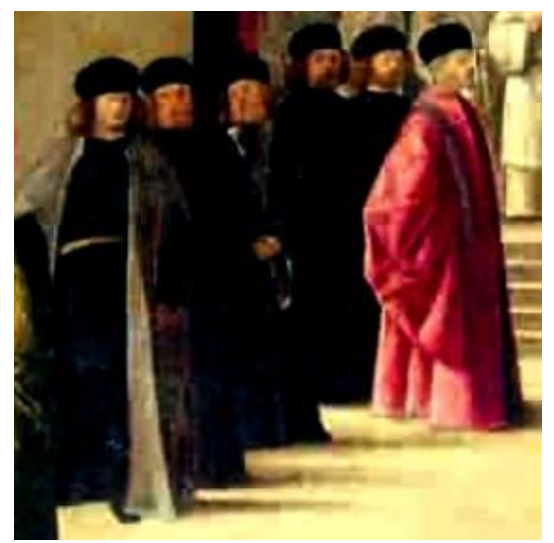

Les deux dynasties mameloukes ont été à l'initiative de nombreux projets monumentaux et architecturaux. A l'arrière-plan, nous remarquons la grande mosquée des Omeyades et ses minarets. Le minaret de Qaitbay, construit en 1488, se situe au troisième plan, à gauche, de l'œuvre. Il est pourvu de cinq fenêtres extérieures et est orné d'éléments propres à l'architecture mamelouke.

Figure 04 : Comparaison entre la peinture et une photographie du minaret du sultan Qaytbây.
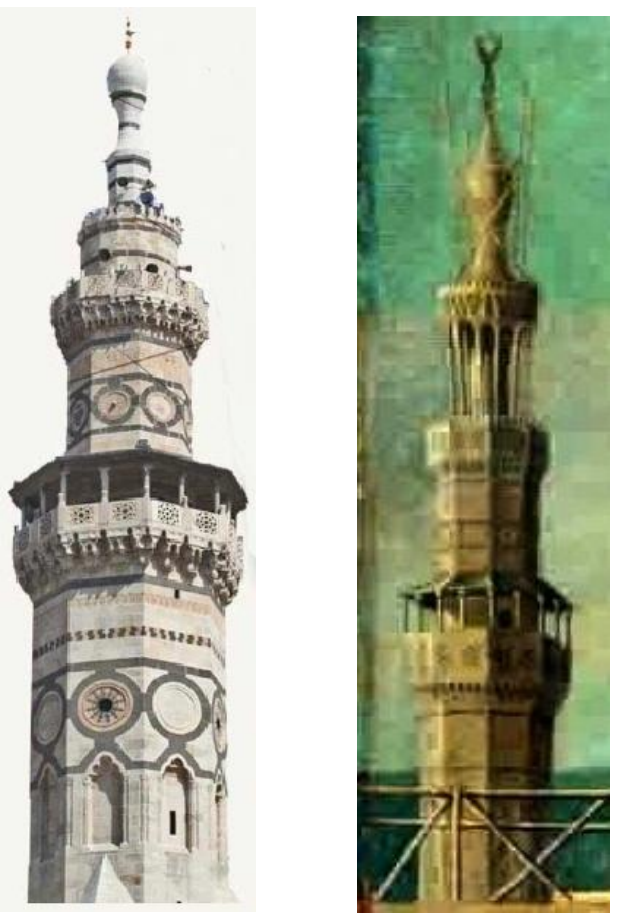

Nous comprenons dès lors, grâce aux indications vestimentaires et architecturales, que la 
scène représentée a pour cadre Damas entre 1488 et 1511, peinte sous le règne d'al-Ghawrî en 1511.

C'est ainsi que les Mamelouks se sont présentés deux siècles et demi durant comme les garants de l'orthodoxie sunnite après le transfert de la capitale califale de Baghdâd vers Le Caire. Ils héritèrent, de ce fait, d'une longue tradition diplomatique des puissantes dynasties qui les ont précédées en Égypte depuis l'avènement de l'Islam. Il s'est installé par la suite au Caire une vie de cour intense, où le cérémonial prit une place prépondérante. Chaque élément du quotidien était soumis à un protocole complexe et minutieux, comme ce fut notamment le cas pour les réceptions d'ambassades où la cour se trouvait au-devant des négociations. L'ambassadeur était nommé par le sultan, et devait représenter les intérêts sultaniens à la cour des souverains les plus puissants du monde connu. Ces délégations étaient accompagnées de cadeaux de prestige qui définissaient le niveau d'appréciation de celui qui les recevait. 
Figure 05 : Tableau supposé de Gentille Bellini représentant « la réception d'une ambassade vénitienne à Damas »

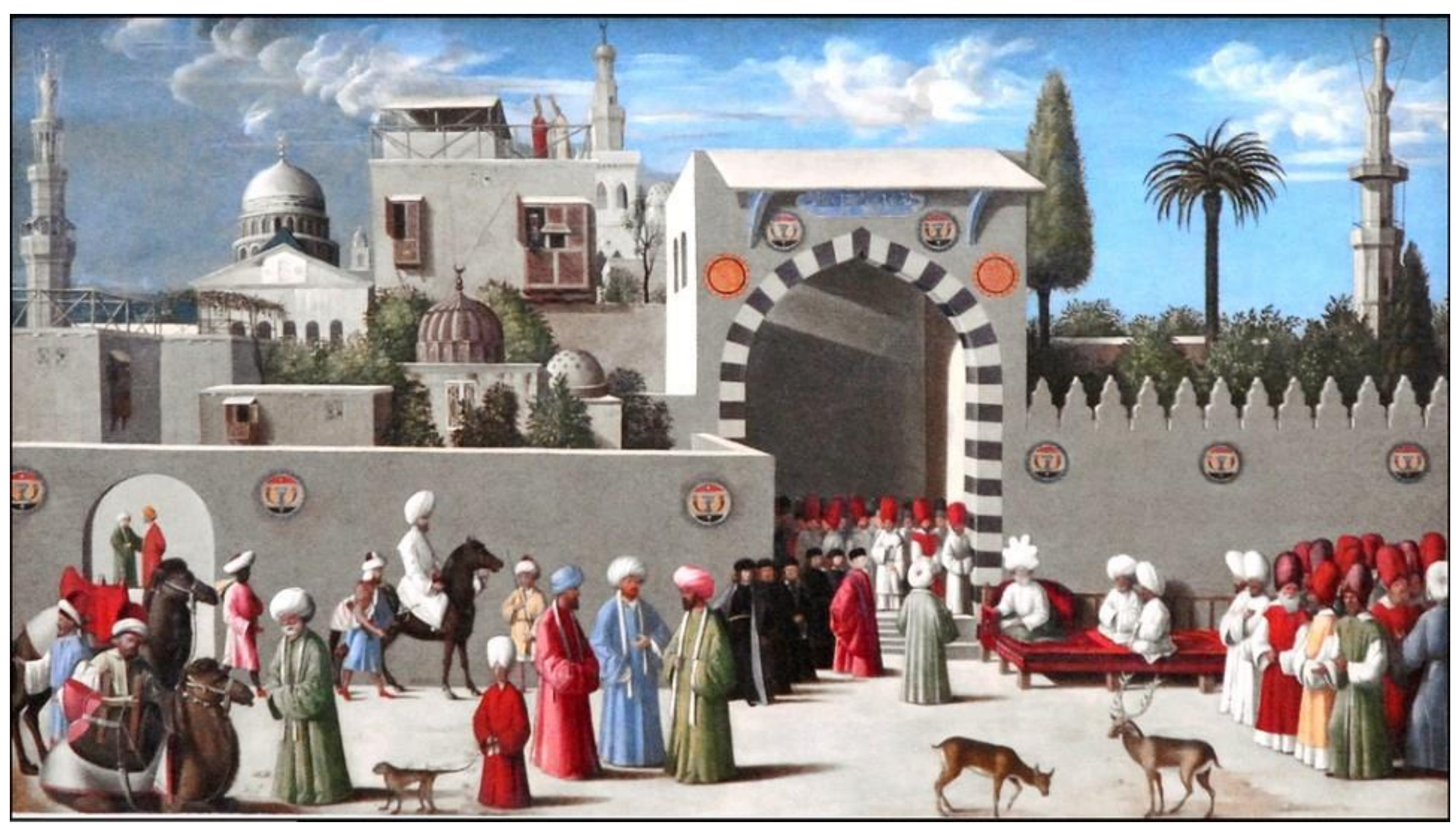


${ }^{1}$ Collectif, op. cit., p. 149-169 passim; IBN IYAS, Journal d'un bourgeois du Caire I, tr. G. Wiet, Tome I, (Paris 1955), 1945, p. 63. [Rabi' I 910] : « La crue du Nil béni atteignit son maximum le 7, qui correspondait au 25 mésori, soit dix-sept jours plus tard que l'année précédente : ce jour-là, le niveau était de 17 coudées et 5 doigts. Le maréchal Qait présida la cérémonie de la rupture de la digue, au milieu d'une grande affluence ; ce fut la dernière fois, car le maréchal allait être très bientôt emprisonné comme nous allons le voir. "

${ }^{2}$ BRESC, op. cit.,p. 84/ IBN IYAS, Tome I, p. 48. [Dhul-qa'da]: «Le ler correspondait au 18 bashans de l'année copte, le sultan quitta sa tenue de laine pour se vêtir de blanc et commença les parties de polo. » Le beige pour l'hivers et le blanc pour l'été. "

${ }^{3}$ CARAYON Agnés, La furussiyya des mamlûks: une élite sociale à cheval, Thèse d'État, Université Aix-Marseille, Aix-en-Provence, 2012, p. 399. ; BRESC, op. cit., p. 84/ IBN IYAS, Tome I, p. 48 (Se référer à la note précédente.)

${ }^{4}$ IBN IYAS, Tome I, p. 37. [Rabi' I 908] : « Le Sultan célébra dans la Cour royale l'anniversaire de la naissance du Prophète, en présence des quatre cadis et des vingt-quatre commandants de mille au grand complet, ce qui ne s'était presque jamais vu. »

${ }^{5}$ Pèlerinage des musulmans à La Mecque, un des cinq piliers de l'Islam. (BRESC, op. cit.,p. 84.)

${ }^{6}$ Paroles et faits relayés dans un recueil selon une chaîne de transmission bien précise. (BRESC, op. cit. p. 84. »)

${ }^{7}$ Savant musulman du IXe siècle à l'origine du plus étendu et connu recueil de hadith ; IBN IYAS, Tome I, p. 229. [Ramadhân 919] : « La lecture complète de Traditions de Bukhârî eut lieu le jeudi 26 sous la grande tente dressée au milieu de la Cour royale, selon l'usage. Assistèrent à cette cérémonie grandioses les quatre cadis, les grands ulémas et les bourses furent attribuées comme d'habitude.»

${ }^{8}$ Pilier de l'islam qui laisse place à un mois de jeûne du lever au coucher du soleil. (Collectif, op. cit., p. 124-125.)

${ }^{9}$ La prière de la fête du sacrifice et la rupture du jeûne se faisaient généralement à l'hippodrome.

${ }^{10}$ La prière du vendredi se faisait à la mosquée de la Citadelle, le discours était prononcé par le calife en personne. La venue du sultan se faisant en grand apparat.

${ }^{11}$ BRESC, op. cit., p. 84

${ }^{12}$ Ibid., p. 84.

${ }^{13}$ Le défilé à cette occasion était parmi les plus importants, l'ensemble de la ville était décoré et remis à neuf. L'ensemble des emblèmes de la souveraineté y était exhibé. De même que des tapis de soie étaient étalés au sol sur l'itinéraire que le sultan devait emprunter, de Bâb al-Nâsr à la Citadelle. Cette pratique était attestée seulement lors du retour d'un très long voyage du sultan.

${ }^{14}$ L'audience des Mazâlim avait lieu dans l'Iwân ou à la maison de justice. "Les grands kidât des quatre rites sont à sa droite, puis viennent les fonctionnaires financiers tandis qu'à sa gauche se trouve le vizir, s'il est homme de plume, puis le Kaâtib al-sirr. Le protocole semble accorder la préséance aux civiles puisque les hommes de sabre sont debout à l'exception des grands émirs de cent qui sont assis derrière le sultan. Le deuxième signe de préséance réside dans le fait que les personnages les plus honorés siègent à droite du souverain. » (CHAPOUTOT, Liens, p. 91.)

${ }^{15}$ Ibid., p 90.

${ }^{16}$ MALAMUT Élisabeth et OUERFELLI Mohamed (dir.), Les échanges en Méditerranée médiévale. Marqueurs, réseaux, circulations, contacts, Aix-

en-Provence, Presses universitaires de Provence, 2012, p. 69.

${ }^{17}$ Ibid., p. 88.

${ }^{18}$ CHAPOUTOT, Liens, p. 91.

${ }^{19}$ La référence étant le traité contracté entre les musulmans de Médine et les Qurayshites de La Mecque (Traité d'al-Hudaybiya signé en 628.)

${ }^{20}$ TALBI Alaa, «Un exemple de diplomatie en temps de guerre entre les Mongols de Perse et les Mamelouks d'Égypte », dans Annales Islamologiques 41, 2007, p. 121.

21 Historien et mathématicien égyptien du XVe siècle.

${ }^{22}$ Théologien et jurisconsulte musulman du XVIIIe siècle, surnommé sheikh al-islâm (=savant de l'islam)

${ }^{23}$ ZOUACHE Abbès, "La guerre dans le monde arabo-musulman médiéval : perspectives anthropologiques. Introduction », dans Annales Islamologiques 43, 2009, p. 17.

${ }^{24}$ ZOUACHE, op. cit., p. 18

${ }^{25}$ Sultan mamelouk, de la dynastie bahrite ayant régné sur le sultanat de 1279 à 1290.

${ }^{26}$ Le terme « harb » est préféré - Ibid., p. 18.

${ }^{27}$ Juriste musulman d'obédience shafi'ite, il endossa plusieurs fois la fonction d'ambassadeur pour les califes abbassides.

${ }^{28}$ OUERFELLI Mohamed, Gouverner en Islam entre le Xe siècle et le XVe siècle. Iraq jusqu'en 1258, Syrie, Hijaz, Yémen, Egypte, Maghreb et alAndalus, Paris, Ellipses, coll. «CAPES/Agrégation», 2014, p. 87.,

${ }^{29}$ AL-MAQRIZI, Histoire des sultans mamlouks de l'Egypte, trad. QUATREMERE, Paris, 1840, t.1, p. 183-185. / Ce discours s'inscrit dans un contexte où le calife abbasside a été rétabli pour légitimer le pouvoir mamelouk, c'est ainsi que le calife prononce ce discours en l'honneur du sultan Baybars en 1262 (OUERFELLI, Gouverner, p. 86.)

30 JAVIER APELLANIZ RUIZ DE GALARRETA Franscisco, «Banquiers, diplomates et pouvoir sultanien. Une affaire d'épices sous les Mamelouks circassiens », dans Annales Islamiques 38, 2004, p. 285.

${ }^{31}=$ Commerçants

${ }^{32}$ Ibid., p. 295.

${ }^{33}$ Savant maghrébin du XVe siècle, il est considéré comme le père de la sociologie.

${ }^{34}$ A noter que certains ambassadeurs n'étaient pas musulmans.

${ }^{35}$ CUTLER Anthony, «Les échanges de dons entre Byzance et l'Islam » dans Journal des savants, Volume 1, Numéro 1, 1996, p. 53.

${ }^{36}$ Il s'agissait d'un système mis en place par les Byzantins pour avertir des événements.

${ }^{37}$ Des individus comme al-Jazarî ont permis des progrès en matière scientifique et notamment dans le domaine mécanique aux XIIème- XIIIème siècles. Il a été rendu populaire par son "Livre de la connaissance des procédés mécaniques" (Kitab fi ma 'rifat al-hiyal al- handasiyya) rédigé en l'an 1206. Certains automates servaient du thé, d'autres jouaient de la musique ou encore donnaient l'heure. Toutes les cours du monde connues s'empressaient d'acquérir cela.

${ }^{38}$ CUTLER Anthony, «Les échanges de dons entre Byzance et l'Islam » dans Journal des savants, Volume 1, Numéro 1, 1996, p. 53

${ }^{39}$ A titre d'exemple, lors d'une visite du sultan Barqûq en Syrie, le gouverneur lui offrit 50 chevaux, 32 juments, 118 chevaux de trait, 4 files de chameaux et 80 chameaux, on chargeait ces animaux de fruits, de sucrerie, de légumes marinés et d'autres aliments, c'est dire l'importance et l'aspect non négligeable de ces offrandes.

${ }^{40}$ Le don pouvait être exposé en jour de fête, la cour était friande de ce genre de décorations (Ibid., p. 52.)

${ }^{41}$ Parmi les exemples de correspondances entre les royaumes chrétiens d'Occident et les royaumes musulmans d'Orient, un fait marqua les esprits.

Une femme qui se présentait comme « Reine des Francs » proposa au calife al-Muktafi (293H/906) une multitude de dons, dont sa main en mariage, à condition de l'aider à repousser les Sarrasins. Il s'agissait probablement de Théodora épouse du sénateur romain Théophylacte. (CUTLER, op. cit., p. 51-52)

${ }^{42}$ Le sceau d'or était surtout le fait des souverains byzantins. Le poids variait selon la stature du bénéficiaire. 
${ }^{43}$ Voici l'exemple d'une évaluation de don à la cour byzantine : « Et puis [après la troisième prostration de l'ambassadeur] les silentiarioi reçoivent tous les cadeaux et ils les livrent, puis se fait l'évaluation des dons. Et les responsables de la vestosakra amènent l'évaluation au magistros pour qu'il sache quelle est [la valeur] de chaque cadeau, de sorte qu'il soit en mesure de rappeler à l'empereur au moment des échanges des cadeaux ce qu'il doit faire renvoyer par ses ambassadeurs. " (Le rituel d'évaluation les dons à la cour byzantine, De ceremoniis, 1 :89, 407, ligne 7-13.) Le De ceremoniis aulæ byzantinæ («Des cérémonies de la cour byzantine ») est le terme latin utilisé pour désigner la compilation des protocoles cérémonials de la cour impériale byzantine.

${ }^{44}$ Cité dans RAGHREB Youssef, Les messagers volants en terre d'Islam, CNRS, Paris, 2002, p. 153/ On appelait cela " écriture de poussière " (= Qalam al-Janâh) du fait de la finesse de la calligraphie. 53 Ibid., p. 167.

${ }^{45}$ Ibid., p. 167.

${ }^{46}$ Ibid., p. 168.

${ }^{47}$ OUERFELLI, Les échanges, p. 72.

${ }^{48} \mathrm{Ce}$ manuel fait état d'accords conclus entre les autorités mameloukes et les principautés franques de Syrie -Palestine. (Ibid., p. 71 .)

${ }^{49}$ Fonctionnaire du XIVème siècle au service des sultans mamelouks.

${ }^{50}$ OUERFELLI, Les échanges, p. 74.

${ }^{51}$ Souverains chrétiens orientaux : L'empereur byzantin, le roi de Géorgie, le roi de Serbie, le roi de Sinope, le roi de Petit-Arménie, le roi de Rhodes, Souverains chrétiens occidentaux : Roi d'Andalousie (Alphonse) et roi de France, le Pape, le gouverneur de Gênes, le gouverneur de Venise, le gouverneur de Naples et les gouverneurs des états d'Espagne

${ }_{52}^{5} \mathrm{~S}$ 'en suit l'ensemble des rois chrétiens d'Orient, des Balkans et d'Espagne.

${ }^{53}$ Figure 05 : La réception d'une ambassade vénitienne à Damas.

${ }^{54}$ SAUVAGET Jean, « Une ancienne représentation de Damas au Musée du Louvre », dans Bulletin d'études orientales II, 1945-1946, p. 01.

${ }^{55}$ VASARI Giorgio, Les Vies des meilleurs peintres, sculpteurs et architectes, édition commentée et traduction sous la direction d'André Chastel, Tome

4, Berger-Levrault, 1984, p. 168.

${ }_{56}^{56}$ Ibid., p. 55 - Il s'agit du septième sultan ottoman (1479-1481).

${ }^{57}$ SCHERFER Charles, « Note sur un tableau du Louvre naguère attribué à Gentille Bellini » dans Gazette des beaux arts, Paris, Aout 1895, p. 20.

${ }^{58}$ THENAUD Jean, Le voyage d'Outremer (Egypte, mont Sinay, Palestine) [par Zaccaria Pagani]; publ. et annot. par Ch. Schefer, p. 87.

${ }^{59}$ DE RICCI Seymour, Description raisonnée des peintures du Louvre: Ecoles étrangères Italie, Espagne, Volume 1, n¹157, Paris, $1913, \mathrm{p} .49$.

${ }^{60}$ SAUVAGET, Une ancienne représentation, p. 05.

${ }^{61}$ Avant dernier sultan mamelouk (1501-1516).

${ }^{62}$ DE RICCI, op. cit., p. 49.

${ }^{63}$ SAUVAGET, Une ancienne représentation, p. 06.

${ }^{64}$ Ibid., p. 6.

${ }^{65}$ Il s'agit d'une relation d'ambassade de mai 1512 de Zaccaria PAGANNI (SCHERFER, op. cit., p. 202-204.)

${ }^{66}$ SAUVAGET, Une ancienne représentation, p. 06.

${ }^{67}$ Terminologie arabe signifiant « Grande Syrie ».

${ }_{68}^{68}$ «Les jambes croisées sont généralement le fait de personnages qui détiennent le pouvoir. » (GARNER, op. cit., Tome I, p. 229.)

${ }^{69}$ Une sorte d'estrade.

${ }^{70}$ La position assise est la même que celle de l'émir, cela peut traduire l'imitation d'un supérieur par un inferieur. Le supérieur est clairement identifié par ses attributs (couvre-chef, position, distance etc.), en reproduisant la position de leur supérieur, les deux cadis manifestent «un accord de soumission » avec celui-ci (Ibid., p.51)

${ }^{71} \mathrm{~L}$ 'individu qui se distingue par son vêtement rouge est positionné de profil, ce qui a pour signification un signe d'infériorité qui contraste avec la position trois quarts des autres personnages, ce qui n'a pas de sens spécifique, puisqu'elle est la position la plus fréquente. (GARNIER, op. cit., Tome I, p. 124.)

${ }_{72}$ S'agit-il d'un ambassadeur ou d'un consul ? La question est posée par SAUVAGET, op. cit., p. 10.

${ }^{73}$ SCHERFER, op. cit., p. 202.

${ }^{74}$ SAUVAGET, op. cit., p. 10.

${ }^{75}$ C'est en 912/1507, qu'al-Ghawrî y aménagea un jardin avec des arbres et des végétaux importés du bilâd al-Shâm, donnant la sensation d'une véritable oasis dans un climat désertique notamment grâce à la bifurcation de l'aqueduc pour alimenter ces plantes. La chronique d'Ibn Iyâs est un bon moyen de s'en rendre compte : (IBN IYAS, Tome I, p. 99. [Sha'bân 912] : "Le sultan reçut de Syrie des arbustes transportés dans des caisses avec leur terre: pommiers de Syrie, poiriers, cognassiers, cerisiers, plants de vigne, ainsi que des plantes odoriférantes, telles que roses blanches, lis, iris, et d'autres produits de la flore syrienne, jusqu'à un cocotier, également empoté. ») 